\title{
Identification of pulse echo impulse responses for multi source transmission
}

\author{
Gran, Fredrik; Jensen, Jørgen Arendt
}

Published in:

Conference Record of the Thirty-Eighth Asilomar Conference on Signals, Systems and Computers

Link to article, DOI:

10.1109/ACSSC.2004.1399113

Publication date:

2004

Document Version

Publisher's PDF, also known as Version of record

Link back to DTU Orbit

Citation (APA):

Gran, F., \& Jensen, J. A. (2004). Identification of pulse echo impulse responses for multi source transmission. In Conference Record of the Thirty-Eighth Asilomar Conference on Signals, Systems and Computers (Vol. 1-2, pp. 168-172). IEEE. Asilomar Conference on Signals, Systems and Computers. Conference Record https://doi.org/10.1109/ACSSC.2004.1399113

\section{General rights}

Copyright and moral rights for the publications made accessible in the public portal are retained by the authors and/or other copyright owners and it is a condition of accessing publications that users recognise and abide by the legal requirements associated with these rights.

- Users may download and print one copy of any publication from the public portal for the purpose of private study or research.

- You may not further distribute the material or use it for any profit-making activity or commercial gain

- You may freely distribute the URL identifying the publication in the public portal 


\title{
Identification of pulse echo impulse responses for multi source transmission
}

\author{
Fredrik Gran and Jørgen Arendt Jensen \\ Center for Fast Ultrasound Imaging, Ørsted•DTU, Bldg. 348, \\ Technical University of Denmark, DK-2800 Kgs. Lyngby, Denmark
}

\begin{abstract}
In this paper a method for acquiring data from several simultaneously transmitting elements in synthetic transmit aperture (STA) ultrasound imaging is proposed. Several transmitters are excited simultaneously using pseudo-random sequences. The received signal at a given time point and receiver is a mixture of the information corresponding to several transmitters. There is, thus, no direct way of determining which information corresponds to which transmitter, preventing proper focusing. In this paper we decode the received signal by estimating the pulse echo impulse responses between every transmitter and receiver pair, using a least squares estimator. The decoding is done instantaneously, making information from several transmitters available after only one transmission. This limits the influence of motion artifacts both in the decoding step and when the STA focusing scheme is applied. The method is evaluated using the simulation tool Field II. Three point spread functions are simulated where axial movement of $1 \mathrm{~m} / \mathrm{s}$ is present. The axial resolution for the moving scatterer is $0.249 \mathrm{~mm}(-3 \mathrm{~dB})$ and $0.291 \mathrm{~mm}(-6 \mathrm{~dB})$, which is compared to a standard STA transmission scheme with sequential excitation of the transmitters using a chirp excitation. The axial resolution was in this case $0.260 \mathrm{~mm}(-3 \mathrm{~dB})$ and $0.611 \mathrm{~mm}(-6 \mathrm{~dB})$. Also a blood vessel is simulated with an angle of $45^{\circ}$ to the acoustic axis with a peak flow of $1 \mathrm{~m} / \mathrm{s}$. The velocity is estimated with a mean bias of $2.57 \%$ and a mean standard deviation of $0.505 \%$ relative to the peak velocity of the flow.
\end{abstract}

\section{INTRODUCTION}

In traditional synthetic transmit aperture imaging (STA) one transmitter is active at a time. All receivers are actively listening for echoes from this specific transmitter. The single transmitter is creating a spherical wave, spreading the acoustic energy in all directions simultaneously. This makes it possible to create several image lines in every transmission. By transmitting several times from different locations on the aperture, the full aperture can be synthesized. This makes it possible to attain perfect dynamic transmit- and receive focusing, assuming constant wave propagation velocity.

The drawbacks of this method are, however, poor signal-tonoise ratio (SNR), due to the use of only one transmitter in every transmission, and the method assumes stationarity for coherent summing of the data to synthesize a large aperture. The problem concerning SNR has been addressed previously in [1-4], where it was suggested to create a high energy virtual source by grouping and focusing several transducer elements close to the transducer surface. This can be achieved either by focusing in front or behind the transducer. It has also been proposed [5-9] to use longer linear frequency modulated signals (chirps) to increase the transmitted energy without increasing the maximum amplitude of the transmitted waveform.

Spatial encoding has also been proposed to increase the transmitted energy and thereby increase the SNR. In $[10,11]$ several transmitters were excited at the same time using an Hadamard encoding scheme. If $N$ transmitters are used simultaneously, $N$ transmissions have to be made for decoding the sequence. Since the decoding is based on several transmissions, tissue motion will degrade the decoding process. In [12] Golay encoding was proposed to spatially encode the transmitters. However, this approach suffers from the same limitations as the Hadamard encoding scheme.

In this paper the latter of the two problems is addressed. A method for spatial encoding using pseudo-random sequences is proposed. This has been suggested previously in $[13,14]$ where pseudo random codes were used to excite several of the transmitters simultaneously. The received signal was then modeled as the weighted sum of the system impulse response for certain scatterer positions. A fixed grid of scatterers is defined and the weights (scatterer strengths) in the image are estimated. In this paper, however, several transmitters are excited simultaneously and are decoded instantaneously at the receiver by estimating the pulse echo impulse response between the transmitters and the receivers. The impulse responses are modeled as FIR filters and no scatter map is defined, which means that no assumptions on the geometry is made. The decoded echoes are then beamformed using a synthetic transmit aperture focusing technique. The principle can be seen in Fig. 1 compared to conventional STA in Fig. 2. The outline of the paper is as follows: in Section II, the theory of the proposed method will be explained and in Section III the beamforming algorithm associated with the method is described. In Section IV a simulation study evaluates the point spread function (PSF) for the proposed method. Additionally a flow simulation is presented where the velocity of the flow is estimated. Finally, concluding remarks are given 


$$
\begin{array}{l|l|}
\text { XMT } & \text { Code } 1 \\
\hline \text { Code } 2 \\
\hline \text { RCV } \\
\cline { 2 - 2 } \\
\cline { 2 - 2 } & \text { Received signal from xmt no: } 1 \\
\hline
\end{array}
$$

\section{Time}

Fig. 1. The principle for the proposed method. Two different transmitters are excited simultaneously, yielding a higher effective pulse repetition frequency and a higher frame rate.

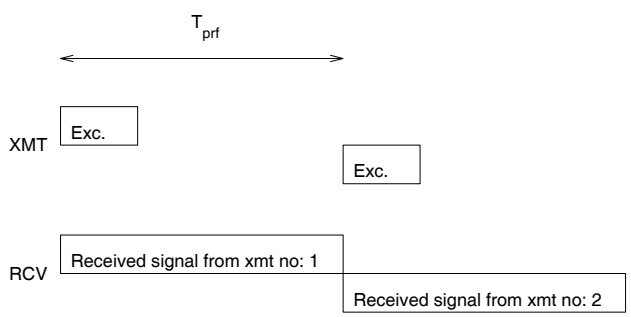

Time

Fig. 2. The conventional synthetic transmit aperture approach. Every transmitter has access to the system separately, giving a lower pulse repetition frequency.

in Section V.

\section{THEORY}

Consider a system with $K$ transmitters and $Q$ receivers. Each transmitter is assigned a specific code $x_{k}(n)$ of length $M$ generated from a white, zero mean process with gaussian probability distribution. It assumed that the system dynamics is linear, and the impulse response between the $k$ :th transmitter and the $q$ :th receiver is modeled by an FIR filter of order $N$. The impulse response between a given transmitter and receiver pair accounts for all phenomena that effect the transmitted waveform seen from the receiver. This impulse response, thus, represents the transducer impulse response, as well as interaction with tissue, scattering and attenuation. The sampled received signal on the $q$ :th receiving element can then be written

$$
y_{q}(n)=\sum_{k=1}^{K} \mathbf{h}_{q k}^{H} \mathbf{x}_{k}(n)+v_{q}(n)
$$

where $\mathbf{h}_{q k}=\left(\begin{array}{llll}h_{q k}^{0} & h_{q k}^{1} & \ldots & h_{q k}^{N-1}\end{array}\right)^{T}$ is the impulse response between the $k$ :th transmitter and the $q$ :th receiver and is assumed to be stationary during the transmission. $\mathbf{A}^{H}$ denotes the complex conjugate transpose of $\mathbf{A}$. The signal vector for transmitter $k$ and time point $n$ is given by $\mathbf{x}_{k}(n)=$ $\left(\begin{array}{llll}x_{k}(n) & x_{k}(n-1) & \ldots & x_{k}(n-N+1)\end{array}\right)^{T}$, and $v_{q}(n)$ is the noise on the $q$ :th receiver, which is assumed to be a white, zero mean, and gaussian distributed process. By writing the received signals on the $Q$ receivers as a vector $\mathbf{y}(n)=$ $\left(\begin{array}{llll}y_{1}(n) & y_{2}(n) & \ldots & y_{Q}(n)\end{array}\right)^{T}$, a more compact notation gives

$$
\mathbf{y}(n)=\mathbf{H} \mathbf{x}(n)+\mathbf{v}(n),
$$

where

$$
\begin{aligned}
& \mathbf{H}=\left(\begin{array}{cccc}
\mathbf{h}_{11}^{H} & \mathbf{h}_{12}^{H} & \ldots & \mathbf{h}_{1 K}^{H} \\
\mathbf{h}_{21}^{H} & \mathbf{h}_{22}^{H} & \ldots & \mathbf{h}_{2 K}^{H} \\
\vdots & \vdots & \ddots & \vdots \\
\mathbf{h}_{Q 1}^{H} & \mathbf{h}_{Q 2}^{H} & \ldots & \mathbf{h}_{Q K}^{H}
\end{array}\right) \\
& \mathbf{x}(n)=\left(\begin{array}{llll}
\mathbf{x}_{1}(n)^{T} & \mathbf{x}_{2}(n)^{T} & \ldots & \mathbf{x}_{K}(n)^{T}
\end{array}\right)^{T}
\end{aligned}
$$

and $\mathbf{v}(n)$ is the noise on all receivers to time-point $n$. The noise on the different receivers are considered to be mutually uncorrelated, such that $E\left[\mathbf{v}(n) \mathbf{v}^{H}(n)\right]=\sigma_{v}^{2} \mathbf{I}^{1}$,where $\sigma_{v}^{2}$ is the variance of the system noise. The (windowed) least squares estimate (LSE) at time point $l$ of the impulse response matrix $\mathbf{H}$ is [15]

$$
\hat{\mathbf{H}}_{L S}(l)=\hat{\mathbf{R}}_{y x}(l) \hat{\mathbf{R}}_{x x}(l)^{-1},
$$

where

$$
\begin{aligned}
\hat{\mathbf{R}}_{x x}(l) & =\sum_{n=l-L+1}^{l} \mathbf{x}(n) \mathbf{x}^{H}(n), \\
\hat{\mathbf{R}}_{y x}(l) & =\sum_{n=l-L+1}^{l} \mathbf{y}(n) \mathbf{x}^{H}(n),
\end{aligned}
$$

are the estimates of the auto correlation matrix of $\mathbf{x}(n)$ and the cross correlation matrix of $\mathbf{x}(n)$ and $\mathbf{y}(n)$, respectively. $L$ determines how many observations are used to determine the estimate of $\mathbf{H}(l) . \quad \hat{\mathbf{R}}_{x x}$ has to have full rank to identify the correct solution to (5). Since the dimensions of $\hat{\mathbf{R}}_{x x}$ are $K N \times$ $K N$, there has to be at least $K N$ linear independent signal vectors available. After one transmission there are $M+N-1$ observations of the process available. This gives the inequality

$$
\begin{aligned}
M+N-1 & \geq K N \\
& \Downarrow \\
M & \geq(K-1) N+1,
\end{aligned}
$$

\footnotetext{
${ }^{1} E[\mathbf{Q}]$ denotes the expectancy value of $\mathbf{Q}$.
} 
if the impulse responses are to be estimated in only one transmission, provided that the signal vectors are linear independent. The $K N$ signal vectors can be made to be linear independent by choosing $K$ different pseudo random sequences satisfying the code length condition given in (8).

\section{BEAMFORMING}

This section describes the beamforming, which has been applied to properly focus the data. By estimating the impulse response vectors $\mathbf{h}_{q k}$, it is in fact known what the received signal on the $q$ :th receiver would look like if only the $k$ :th transmitter was active and transmitting a delta function. This allows the synthetic aperture focusing technique described in [16] to be applied.

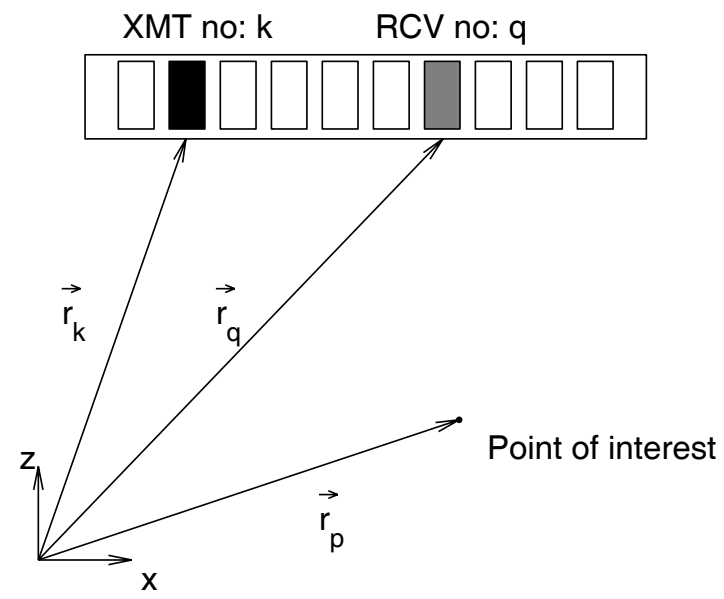

Fig. 3. Principles of synthetic aperture focusing for a point $p$ for the $k$ :th transmitter and the $q$ :th receiver.

Since the system has access to defocused transmissions from all transmitters on the aperture, it is possible to focus in any arbitrarily chosen point of interest. The amplitude in a point $p$ in the image is given by

$$
A\left(\vec{r}_{p}\right)=\sum_{k=1}^{K} \sum_{q=1}^{Q} g_{q} h_{q k}\left(t_{p q k}\right),
$$

where $g_{q}$ is a weighting function (apodization) over the receiving aperture, and $h_{q k}(t)$ is the time signal corresponding to the identified impulse response between the $q$ :th receiver and the $k$ :th transmitter. Here $t_{p q k}$ is the time corresponding to the geometrical travel distance from the $k$ :th transmitter to the point $p$ and back to the $q$ :th receiver:

$$
t_{p q k}=\frac{\left\|\vec{r}_{p}-\vec{r}_{k}\right\|+\left\|\vec{r}_{q}-\vec{r}_{p}\right\|}{c},
$$

where $c$ is the speed of sound, and $\vec{r}_{p}-\vec{r}_{k}$ is the vector from the transmitter to the point $p$, and $\vec{r}_{q}-\vec{r}_{p}$ is the vector from the point $p$ to the receiver as indicated in Fig. 3. Summing focused contributions from all transmitters and transmissions for all receiving elements, gives both dynamic transmit and receive focusing. In this paper the concept of virtual sources is applied. The virtual sources are created by grouping a number of transducer elements and focusing close to the transducer surface [5]. In this way a high energy defocused transmitter is created. In this paper, however, the focusing is made in front of the actual transducer surface. Each virtual source is considered as a single transmitter, and assigned an individual code. Since the virtual source is treated as a single transmitter, a time (depth) compensation has to be applied in the beamforming, to compensate for the displacement of the virtual source compared to the transducer surface.

\section{Simulations}

In this section the proposed method is evaluated using simulations. The simulations are based on the simulation software Field II [17, 18]. In Section IV-A the PSF will be evaluated in three points, where the second point exhibits an axial movement of $1 \mathrm{~m} / \mathrm{s}$. In Section IV-B the method will be evaluated by simulating a blood vessel with a parabolic flow profile, where the flow profile is estimated.

\section{A. Point spread function}

A $7 \mathrm{MHz}, 128$ element transducer was simulated. The kerf was $0.035 \mathrm{~mm}$, pitch $0.208 \mathrm{~mm}$ and height $4.5 \mathrm{~mm}$. The transmitting aperture was created by grouping and focusing 64 of the central transducer elements, so that 4 virtual sources were formed. The reason for using only the 64 central elements was to minimize the spacing between the virtual sources, to reduce the effect of grating lobes. Each virtual source was created using 16 transducer elements, which were focused $2.5 \mathrm{~mm}$ in front of the transducer surface. The receiving aperture used all 128 elements with a Hanning weighting. The simulation sampling frequency was $120 \mathrm{MHz}$, but the output was downsampled to $40 \mathrm{MHz}$ to reduce the amount of data. The transducer impulse response was set to be two cycles of a sinusoid at $7 \mathrm{MHz}$ with a temporal Hanning weighting. Two different pseudo random sequences were generated by the Matlab function randn with a duration of $32.5 \mu \mathrm{s}$. This enabled the decoding of two simultaneously transmitting virtual sources, which implied that only two virtual sources could be active at the same time. The two outer most transmitters were excited and decoded in the first transmission, where after the two inner most transmitters were excited and decoded. In this way two low resolution images ${ }^{2}$ could be formed in every transmission,

\footnotetext{
${ }^{2}$ The term low resolution image represents the beamformed echoes from only one single transmitter, by summing beamformed data from several transmitters a large aperture can be synthesized creating a high resolution image $[16]$
} 
which enables fully coherent summing of the two low resolution images. The pulse repetition frequency was set to $8 \mathrm{kHz}$. Three scatterers were positioned directly below the transducer at depths 30, 40 and $50 \mathrm{~mm}$. The second scatterer was moved with a constant velocity of $1 \mathrm{~m} / \mathrm{s}$ away from the transducer. The resulting PSF's can be seen in Fig. 5. The reference image was created using the same transducer setup, but the transmitters were excited separately. The excitation waveform was a $20 \mu \mathrm{s}$ chirp with a center frequency of $7 \mathrm{MHz}$ with a $75 \%$ bandwidth. The waveform was tapered using a Tukey window with $10 \%$ tapering. The compression filter was the time reversed excitation waveform, mismatched using a Chebyshev window with a relative attenuation of $70 \mathrm{~dB}$. The mismatching was done to suppress axial sidelobes [9]. The resolution for the proposed method was for the moving scatterer: axially $0.249 \mathrm{~mm}(-3 \mathrm{~dB})$, $0.291 \mathrm{~mm}(-6 \mathrm{~dB})$ and laterally $0.521 \mathrm{~mm}(-3 \mathrm{~dB}), 0.748 \mathrm{~mm}(-$ $6 \mathrm{~dB})$ and the lateral sidlobe level was below $-45 \mathrm{~dB}$. The corresponding resolution for the reference method was: axially $0.260 \mathrm{~mm}(-3 \mathrm{~dB}), 0.611 \mathrm{~mm}(-6 \mathrm{~dB})$ and laterally $0.489 \mathrm{~mm}(-$ $3 \mathrm{~dB}), 0.643 \mathrm{~mm}(-6 \mathrm{~dB})$ and the lateral sidlobe level was below $-45 \mathrm{~dB}$.

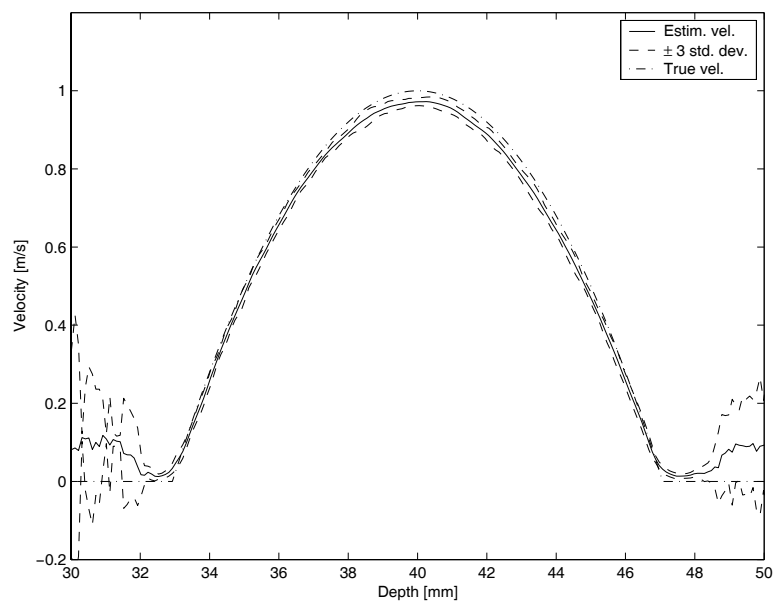

Fig. 4. The estimated flow as a function of depth. The dash dotted line indicates the true flow profile with a peak velocity of $1 \mathrm{~m} / \mathrm{s}$. The solid line indicates the mean estimated profile. The dashed line shows the interval of \pm 3 standard deviation of the estimated flow profile

\section{B. Blood flow estimation}

A flow simulation was also performed using Field II. The same transducer setup and excitation was used as in Section IV-A. The vessel was positioned at a depth of $40 \mathrm{~mm}$ with a diameter of $10 \mathrm{~mm}$. The flow profile was parabolic with a peak velocity of $1 \mathrm{~m} / \mathrm{s}$. The angle between the flow and the acoustic axis was $45^{\circ}$. Since it has been shown previously that it is possible to automatically detect the angle of the flow [19], it was assumed to be known. The beamforming described in Section
III was applied to beamform image lines in the direction of the flow. The lateral spatial sampling frequency corresponded to $\frac{\lambda}{40}$. Lines beamformed along the flow direction from one given image were correlated with the corresponding lines from the previous image. The length of the lines were $20 \lambda$. The cross correlation of the lines were used to find the velocity of the flow $[19,20]$. The shift in the peak in the cross correlation function indicates the spatial shift of the speckle pattern between two images. Thus, dividing by the time for acquiring the two images yields the velocity estimate. To further improve the velocity estimate, 10 successive cross-correlations were averaged. The estimated velocity as function of depth can be seen in Fig. 4. The mean bias was $2.57 \%$ and the mean relative standard deviation was $0.505 \%$. The mean profile and the standard deviation was found from 20 successive velocity estimates. The results are comparable to those found in [20].

\section{CONCLUSiON}

In this paper a method for making the image acquisition faster has been proposed. It is possible to decode the information originating from different transmitters instantaneously, which means that the image can be created faster. The proposed method has been analyzed in simulations of the PSF, where movement of $1 \mathrm{~m} / \mathrm{s}$ was present. A flow simulation has also been performed with a vessel with an angle of $45^{\circ}$ to the acoustic axis. The velocity was estimated with a mean bias of $2.57 \%$ and a mean standard deviation of $0.505 \%$. Future work will include

- Impact on signal to noise ratio for the proposed method.

- Feasibility in a physical system.

- Relaxation of the demand on code length

\section{ACKNOWLEDGMENT}

This work was supported by grant 9700883,9700563 and 26-01-0178 from the Danish Science Foundation and by B-K Medical A/S, Denmark.

\section{REFERENCES}

[1] M. O’Donnell and L. J. Thomas, "Efficient synthetic aperture imaging from a circular aperture with possible application to catheter-based imaging," IEEE Trans. Ultrason., Ferroelec., Freq. Contr., vol. 39, pp. 366-380, 1992.

[2] M. Karaman, P. C. Li, and M. O’Donnell, "Synthetic aperture imaging for small scale systems," IEEE Trans. Ultrason., Ferroelec., Freq. Contr., vol. 42, pp. 429-442, 1995.

[3] C. Passmann and H. Ermert, "A 100-MHz ultrasound imaging system for dermatologic and ophthalmologic diagnostics," IEEE Trans. Ultrason., Ferroelec., Freq. Contr., vol. 43, pp. 545-552, 1996.

[4] C. H. Frazier and W. D. O'Brien, "Synthetic aperture techniques with a virtual source element," IEEE Trans. Ultrason., Ferroelec., Freq. Contr. vol. 45, pp. 196-207, 1998.

[5] M. O'Donnell, "Coded excitation system for improving the penetration of real-time phased-array imaging systems," IEEE Trans. Ultrason., Ferroelec., Freq. Contr., vol. 39, pp. 341-351, 1992. 

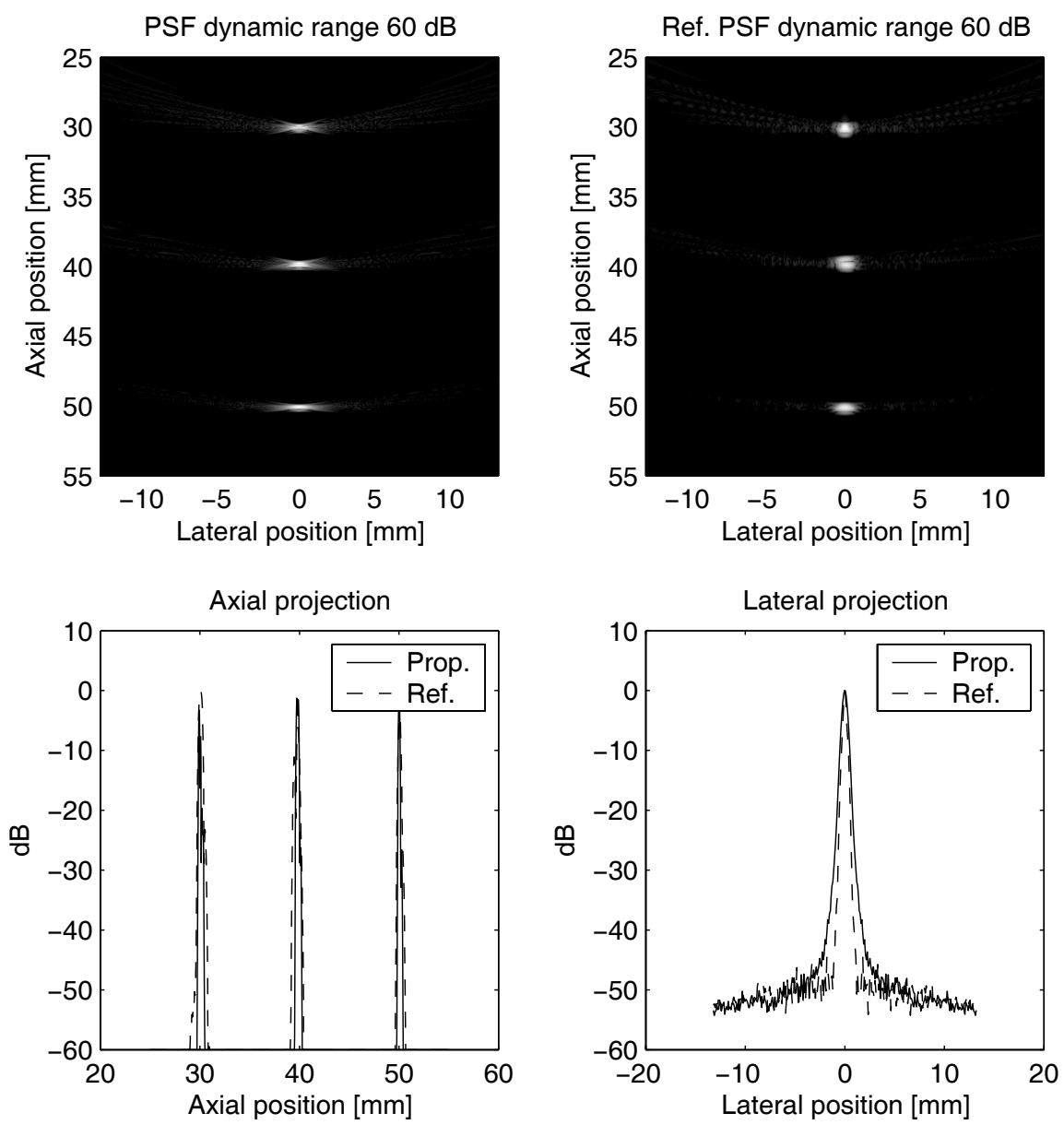

Fig. 5. The PSF's of the proposed method at depths 30,40 and $50 \mathrm{~mm}$ can be seen in the left top figure, whereas the PSF's of the reference method can be seen in the right top figure. The axial an lateral projections can be seen in the left and right bottom figures respectively. (Solid line indicates proposed method, dashed line indicates reference method) The second scatterer is moving with a velocity of $1 \mathrm{~m} / \mathrm{s}$.

[6] T. X. Misaridis, K. Gammelmark, C. H. Jørgensen, N. Lindberg, A. H. Thomsen, M. H. Pedersen, and J. A. Jensen, "Potential of coded excitation in medical ultrasound imaging," Ultrasonics, vol. 38, pp. 183-189, 2000 .

[7] T. X. Misaridis, M. H. Pedersen, and J. A. Jensen, "Clinical use and evaluation of coded excitation in B-mode images," in Proc. IEEE Ultrason. Symp., 2000, vol. 2, pp. 1689-1693.

[8] T. X. Misaridis and J. A. Jensen, "An effective coded excitation scheme based on a predistorted FM signal and an optimized digital filter," in Proc. IEEE Ultrason. Symp., 1999, vol. 2, pp. 1589-1593.

[9] T. Misaridis, Ultrasound imaging using coded signals, Ph.D. thesis, Ørsted•DTU, Technical University of Denmark, Lyngby, Denmark, 2001.

[10] R. Y. Chiao, L. J. Thomas, and S. D. Silverstein, "Sparse array imaging with spatially-encoded transmits," in Proc. IEEE Ultrason. Symp., 1997, pp. 1679-1682.

[11] T. Misaridis and J. A. Jensen, "Space-time encoding for high frame rate ultrasound imaging," Ultrasonics, vol. 40, pp. 593-597, 2002.

[12] R. Y. Chiao and L. J. Thomas, "Synthetic transmit aperture using orthogonal golay coded excitation," in Proc. IEEE Ultrason. Symp., 2000, pp. 1469-1472.

[13] Jian Shen and Emad S. Ebbini, "A new coded-excitation ultrasound imaging system-part 1: Basic principles," IEEE Trans. Ultrason., Ferroelec., Freq. Contr., vol. 43, no. 1, pp. 131-140, 1996.

[14] Jian Shen and Emad S. Ebbini, "A new coded-excitation ultrasound imaging system-part 2: Operator design," IEEE Trans. Ultrason., Ferroelec., Freq. Contr., vol. 43, no. 1, pp. 141-148, 1996.

[15] S. Haykin, Adaptive Filter Theory, Prentice-Hall, Englewood Cliffs, New Jersey, 1986.

[16] S. I. Nikolov, Synthetic aperture tissue and flow ultrasound imaging, Ph.D. thesis, Ørsted•DTU, Technical University of Denmark, 2800, Lyngby, Denmark, 2001.

[17] J. A. Jensen and N. B. Svendsen, "Calculation of pressure fields from arbitrarily shaped, apodized, and excited ultrasound transducers," IEEE Trans. Ultrason., Ferroelec., Freq. Contr., vol. 39, pp. 262-267, 1992.

[18] J. A. Jensen, "Field: A program for simulating ultrasound systems," Med. Biol. Eng. Comp., vol. 10th Nordic-Baltic Conference on Biomedical Imaging, Vol. 4, Supplement 1, Part 1, pp. 351-353, 1996 b.

[19] J. A. Jensen, "Velocity vector estimation in synthetic aperture flow and Bmode imaging," in IEEE International Symposium on Biomedical imaging from nano to macro, 2004, pp. 32-35.

[20] J. A. Jensen and S. I. Nikolov, "Directional synthetic aperture flow imaging," IEEE Trans. Ultrason., Ferroelec., Freq. Contr., pp. 1107-1118, 2004. 